\title{
PREVALENCIA DE PARÁSITOS INTESTINALES EN GALLOS DE PELEA DE LA CIUDAD DE CORO, ESTADO FALCÓN, VENEZUELA
}

\author{
Prevalence of Intestinal Parasites in Fighting Cocks of Coro City, \\ Falcon State, Venezuela
}

Dalmiro Cazorla P. ${ }^{1,2}$, Pedro Morales M. ${ }^{1}$

\section{Resumen}

En Venezuela, las peleas de gallos (Gallus gallus domesticus) representan una actividad recreacional importante y una fuente de empleo; sin embargo, su desempeño combativo puede ser afectado por los parásitos gastrointestinales. Se realizó un estudio epidemiológico en el último trimestre de 2012 y primero de 2013 para determinar la prevalencia de parásitos gastrointestinales en gallos de pelea de la ciudad de Coro, estado Falcón, Venezuela. Las muestras $(n=102)$ se analizaron mediante cuatro métodos coprológicos: directo, flotación de Willis-Molloy y Faust y sedimentación en tubo. Se detectó una o más especies de helmintos o protozoarios en 38 aves (37.3\%), habiendo monoparasitismo en el $8.8 \%$ de los casos e infecciones múltiples hasta con parásitos de seis especies. Strongyloides spp (20.\%), Capillaria spp (16.7\%), Choanotaenia spp $(12.8 \%)$ y Heterakis spp (6.9\%) fueron los enteroparásitos más frecuentes. La frecuencia de limpieza de las jaulas ( $\mathrm{p}=0.009)$, el tipo de comederos y bebederos $(\mathrm{p}=0.01)$, el desinfectante empleado para su lavado $(\mathrm{p}=0.03)$, la geofagia $(\mathrm{p}=0.007)$, la frecuencia del tratamiento $(\mathrm{p}=0.009)$ y quien lo prescribe (Médico Veterinario o criador) $(\mathrm{p}=0.0000)$ fueron potenciales factores de riesgo en la dinámica de transmisión y mantenimiento endémico del parasitismo intestinal en los gallos de pelea.

Palabras clave: gallos de pelea, parásitos intestinales, prevalencia, Venezuela

\section{AbSTRACT}

Cockfighting is an important and popular pastime that generates employment opportunities in Venezuela; however, fighting performance can be affected by gastrointestinal parasites. An epidemiological study was carried out during the last trimester of 2012 and first trimester of 2013 to determine the prevalence of gastrointestinal

\footnotetext{
${ }^{1}$ Laboratorio de Entomología, Parasitología y Medicina Tropical (L.E.P.A.M.E.T.), Centro de Investigaciones Biomédicas (C.I.B.), Universidad Nacional Experimental «Francisco de Miranda»(UNEFM), Estado Falcón, Venezuela

${ }^{2}$ E-mail: lutzomyia@hotmail.com
}

Recibido: 22 de mayo de 2013

Aceptado para publicación: 30 de julio de 2013 
parasites in fighting cocks (Gallus gallus domesticus) $(\mathrm{n}=102)$ in the city of Coro, Falcon State, Venezuela. Faecal samples were analysed by direct test, Willis-Molloy $(\mathrm{NaCl})$ and Faust (zinc sulphate) flotation methods and spontaneous sedimentation in tube technique. One or more intestinal protozoa or helminths were observed in 38 roosters $(37.3 \%)$. Monoparasitism was observed in $8.8 \%$ cases and multiparasitism, including up to six parasite species occurred in some individuals. Strongyloides spp (20.6\%), Capillaria spp (16.7\%), Choanotaenia spp (12.8\%), and Heterakis spp (6.9\%) were the most prevalent enteric parasites. The cage cleaning frequency $(\mathrm{p}=0.009)$, type of feeder and drinker $(\mathrm{p}=0.01)$, disinfectant used $(\mathrm{p}=0.03)$, geophagy $(\mathrm{p}=0.007)$, frequency of treatment $(\mathrm{p}=0.009)$ and who prescribe it (veterinarian or bird owner $)(\mathrm{p}=0.0000)$ were potential risk factors related to the transmission and endemic maintenance of intestinal parasitism in fighting cocks.

Key words: fighting cocks, intestinal parasites, prevalence, Venezuela

\section{INTRODUCCIÓN}

En Venezuela, la pelea o lidia de gallos (Gallus gallus domesticus) es una actividad recreativa muy popular y ampliamente extendida que se practica en recintos (clubes gallísticos) denominados comúnmente «galleras». Usualmente, las peleas de gallos se llevan a cabo durante los días de fiesta, fechas patrias o los fines de semana, y se ha convertido en una industria pujante y en crecimiento, que provee numerosos empleos directos e indirectos, especialmente durante las festividades (Ibarra-Velarde et al., 2011).

El criador de gallos de pelea debe ganar las lidias de la manera más expedita posible, contando con animales de excelente conformación fenotípica y genética; es decir, de espíritu combativo, buena conformación, ligero, astuto, decidido y certero, además de plumaje resaltante y colorido (Varela, 2007). Sin embargo, uno de los inconvenientes que afectan el desempeño de estas aves es la pérdida de condición corporal por anorexia, pérdida de sangre y proteínas plasmáticas por el tracto gastrointestinal, alteraciones en el metabolismo proteico, depresión en la actividad de enzimas intestinales y diarrea (Luka y Ndams, 2007; Álvarez et al., 2011; Ogbaje et al., 2012). Estas patologías pueden ser causadas por agentes infecciosos, incluyendo parásitos intestinales de tipo helmintos (nematodos: Tetrameres americana, Sheilospirura hamolusa, Heterakis spp, Ascaridia galli, Strongyloides avium; y platelmintos: Raillietina terragona, Amebotaenia cuneata, Chanotaenia infundibulum) y protozoarios (Eimeria spp) (Permin et al., 1997a; Rodríguez et al., 2001; Luna-Olivares et al., 2006; Ogbaje et al., 2012).

Las aves se pueden infectar a través de los alimentos, agua y suelo (Oniye et al., 2000; Álvarez et al., 2011; Ogbaje et al., 2012). En el caso de las aves de pelea, los criadores permiten que estén al pastoreo, donde obtienen pasto verde, pero además pueden alimentarse con invertebrados, incluyendo artrópodos (insectos), moluscos (babosas, caracoles) y anélidos (lombrices de tierra), quienes pueden ser hospedadores intermediarios o paraténicos de helmintos y protozoos (Oniye et al., 2000; Varela, 2007; Álvarez et al., 2011; Ogbaje et al., 2012). Estos parásitos pueden eventualmente causar enfermedad e incluso la muerte de las aves (Luka y Ndams, 2007).

El control de las enfermedades parasitarias, al igual que otras etiologías, depende en gran medida del diagnóstico preciso y oportuno de los agentes etiológicos, a fin de establecer el correcto y eficaz tratamiento que permita mejorar la rentabilidad de la granja (Varela, 2007; Álvarez et al., 2011). En base 
a esto, el objetivo del presente trabajo fue determinar las especies y prevalencia de protozoarios y helmintos intestinales en gallos de pelea mantenidos en criaderos de la ciudad de Coro, Falcón, Venezuela. Asimismo, se indagó sobre los posibles factores de riesgo que influyen en la adquisición y frecuencia de los parásitos gastrointestinales en la población de gallos de pelea.

\section{Materiales y Métodos}

El estudio se llevó a cabo entre septiembre de 2012 y marzo de 2013, en la ciudad de Coro, municipio Miranda, capital del estado Falcón. La región posee una zona bioclimática del tipo Monte Espinoso Tropical (MET), con clima semiárido y vegetación xerofítica, suelos francamente erosionados sujetos a la acción constante de los vientos alisios, con escasas y erráticas precipitaciones con promedios anuales de 250 a $500 \mathrm{~mm}$, temperatura media anual de 28 $29{ }^{\circ} \mathrm{C}$ y una elevada evapotranspiración (Ewel et al., 1976).

Se hizo contacto con seis establecimientos dedicados a la cría y entrenamiento de gallos de pelea, y cuatro de ellos aceptaron participar en el estudio, lo cual permitió tomar muestras de heces a la totalidad de las aves con edades de 6 a 36 meses $(n=102)$.

Las muestras fueron recolectadas por los dueños en horas matutinas en envases estériles herméticos y se transportaron al laboratorio de parasitología del Centro de Investigaciones Biomédicas (CIB) de la Universidad Nacional Experimental «Francisco de Miranda»(UNEFM). Las muestras, de no ser analizados el día de la toma, se preservaron y fijaron añadiéndoles $4-5 \mathrm{ml}$ de formaldehído al $10 \%$.

Las muestras fueron procesadas por el método coprológico directo y por las técnicas de flotación simple de Willis-Molloy con solución sobresaturada de cloruro de sodio $(\mathrm{NaCl})$, flotación-centrifugación de Faust con sulfato de zinc y de sedimentación en tubo (TSET) (Morales y Pino de Morales, 1977; Botero y Restrepo, 2003). Las muestras fueron observadas por duplicado bajo microscopio de luz (Axiostar Plus, Carl Zeiss, Alemania) con y sin solución yodada de lugol. La identificación de las especies parasitarias se realizó mediante morfología externa e interna comparada de quistes-ooquistes/ trofozoitos y huevos/larvas (Kaufmann, 1996). Los animales se consideraron parasitados cuando se detectó, por lo menos, un estadio de protozoarios (quiste-ooquiste/trofozoito) o helmintos (huevo/larva).

A cada dueño o criador se le entregó una encuesta para indagar sobre aspectos y características de las aves incluyendo tipo de alimentación, condiciones sanitarias de cría, mantenimiento y entrenamiento, asesoría veterinaria y aplicación de antiparasitarios. En base a las respuestas se analizaron las variables tipo de piso (tierra, cemento), suministro de agua (por tubería, por tanque/tonel), frecuencia de limpieza de las jaulas (diario, 1-2 veces por semana), tipo de comederos y bebederos (comercial, artesanal), frecuencia de lavado de comederos y bebederos (diario, 23 veces por semana), tipo de desinfectante empleado (cloro, creolina), tiempo de pastoreo (10 a 30, 31 a 60 minutos), frecuencia de pastoreo (diario, 1-2 veces por semana), geofagia (sí, no), consumo de lombriz de tierra y de insectos (sí, no), tratamiento antiparasitario (sí, no), frecuencia de tratamiento (cada 21-30 días, >30 días), y quien lo prescribe (Médico Veterinario, criador).

La afinidad entre pares de especies que co-existieron se midió mediante el Índice de Fager $\left(\mathrm{I}_{\mathrm{A}, \mathrm{B}}\right)$, determinándose su significancia con la prueba de «t», considerándose que existe afinidad real entre las especies involucradas cuando el valor de «t» calculado fue superior a $1.645(\alpha=5 \%)$ (Morales y Pino de Morales, 1995). La asociación univariante entre los posibles factores de riesgo con la presencia de parásitos intestinales se hizo mediante las pruebas de Ji cuadrado (x2) y x2 de Mantel-Haenszel. En los casos 
donde se encontró una asociación significativa $(\mathrm{p}<0.05)$ en el análisis univariado, la fuerza se determinó mediante los Odds Ratio (OR) por regresión logística múltiple, calculándose su bondad de ajuste con la prueba de HosmerLemeshow, a fin de poder controlar las variables de confusión (confounding variables). Los datos se analizaron con el paquete estadístico Statistix v. 1.0.

\section{Resultados y Discusión}

El 37.3\% de las aves presentó uno o más taxones de helmintos o protozoarios en sus tractos digestivos (Cuadro 1).

Dentro de los helmintos, los nematodos Strongyloides spp (20.6\%) y Capillaria spp (16.7\%) y el cestode Choanotaenia spp (12.8\%) fueron los de mayor prevalencia, mientras que Eimeria spp (4.9\%) fue el único protozoario detectado (Cuadro 1; Figs. 1

Cuadro 1. Prevalencia de parásitos intestinales en gallos de pelea $(n=102)$ de granjas de la ciudad de Coro, estado Falcón, Venezuela (2012-2013)

\begin{tabular}{lrr}
\hline \multirow{2}{*}{ Taxón } & \multicolumn{2}{c}{$\begin{array}{c}\text { Aves } \\
\text { parasitadas }\end{array}$} \\
\cline { 2 - 3 } & \multicolumn{1}{c}{$\%^{1}$} \\
\hline Protozoarios & 5 & 4.9 \\
$\quad$ Eimeria spp & & \\
Helmintos & 21 & 20.6 \\
$\quad$ Strongyloides spp & 17 & 16.7 \\
Capillaria spp & 6 & 5.9 \\
Ascaridia galli & 7 & 6.9 \\
Heterakis spp & 4 & 3.9 \\
Trichostrongylus spp & 13 & 12.8 \\
Choanotaenia spp & 3 & 2.9 \\
Hymenolepis spp & 2 & 2.0 \\
Syngamus spp & 4 & 3.9 \\
Tetrameres spp & 5 & 4.9 \\
Raillietina spp &
\end{tabular}

${ }^{1}$ Los porcentajes se calcularon mediante la división del número de aves infectadas con $1,2,3,4,5$ o 6 parásitos entre el número total $(n=102)$ de aves y 2). El monoparasitismo se presentó en el $8.8 \%$ (9/102) de los casos con predominio de Strongyloides spp (10.5\%), mientras que infecciones múltiples ocurrieron hasta con seis taxones (Cuadro 2).

El índice de Fager $\left(\mathrm{I}_{\mathrm{A}, \mathrm{B}}\right)$ solo reveló afinidades significativas entre Strongyloides $\mathrm{spp}$ con Capillaria spp $\left(\mathrm{I}_{\mathrm{A}, \mathrm{B}}=0.29, \mathrm{t}=1.78\right)$, Choanotaenia $\operatorname{spp}\left(\mathrm{I}_{\mathrm{A}, \mathrm{B}}=0.27, \mathrm{t}=5.12\right) \mathrm{e}$ Hymenolepis spp $\left(\mathrm{I}_{\mathrm{A}, \mathrm{B}}=0.27, \mathrm{t}=4.14\right)$; Capillaria spp con Eimeria $\operatorname{spp}\left(\mathrm{I}_{\mathrm{A}, \mathrm{B}}=0.77\right.$, $\mathrm{t}=6.68)$ y Heterakis spp $\left(\mathrm{I}_{\mathrm{A}, \mathrm{B}}=0.46, \mathrm{t}=7.64\right)$; y Choanotaenia spp con Eimeria spp $\left(\mathrm{I}_{\mathrm{A}, \mathrm{B}}=0.57, \mathrm{t}=2.39\right)$.

Dentro de las características de mantenimiento e higiene de las jaulas, se encontraron asociaciones significativas entre parasitismo intestinal y frecuencia de su limpieza [OR=2.19 (1.11 - 6.47, intervalo de confianza al $95 \%)]$ y tipos de bebederos y comederos $[\mathrm{OR}=2.11(1.11-6.53)]$ así como el desinfectante empleado [OR=2.01 (0.89-5.25)] (Cuadro 3); mientras que con los aspectos relacionados con la alimentación, solo la geofagia $[\mathrm{OR}=2.17(1.13-7.12)]$ mostró una asociación significativa (Cuadro 4).

La frecuencia de administración del antiparasitario [OR=2.19 $(1.11-6.47)] \mathrm{y}$ quien lo prescribe [OR=10.25 (2.15 - 55.32] fueron, asimismo, características asociadas significativamente con el parasitismo gastrointestinal en gallos de pelea (Cuadro 5). Por otro lado, la prueba de Hosmer y Lemeshow mostró valores no significativos $(x 2=5.04 ; \mathrm{p}=0.7785)$, con lo cual se considera que el modelo utilizado cuenta con buen ajuste.

La frecuencia y prevalencia de parásitos intestinales que afectan a G. g. domesticus pueden variar por factores climáticos, ecogeográficos, culturales y técnicas de diagnóstico, así como también por las prácticas higiénico-sanitarias y condiciones de manejo de las granjas (Permin et al., 1997b; Eslami et $a l ., 2009)$. La literatura científica no es muy prolífica en trabajos relacionados con la prevalencia de parásitos gastrointestinales en 
aves de pelea. El $37.3 \%$ de prevalencia encontrada en el presente estudio es menor que el 64\% de prevalencias reportadas en aves de corral en Dinamarca (Chadfield et al., 2001), $73 \%$ en gallinas en Bangladesh (Paul et al., 2012), 64\% en pollos de engorde y gallinas ponedoras en Nigeria (Ogbaje $e t$ al., 2012), 91\% en pollos en Etiopía (Eshetu et $a l ., 2001)$ y $66 \%$ en gallinas en Brasil (Gomes et al., 2009).

Los estudios realizados en Venezuela sobre el tema de parasitismo gastrointestinal en aves son bastante antiguos (Mayoudon, 1955; Mayoudon y Montoya, 1970), mientras que los más recientes se han enfocado básicamente a la problemática de la eimeriosis aviar (Martínez y Bohórquez, 1994; Tamasaukas et al., 1998; Brown et al., 2006). No obstante, los trabajos existentes señalan prevalencias de parasitismo gastrointestinal de 71 y $73 \%$ en gallinas de traspatio en los estados Aragua (Benjoya, 1972) y Falcón (Jiménez y Pereira, 2012), respectivamente, y de $40 \%$ en aves de pelea en el municipio Miranda del estado Falcón (Duno y Sambrano, 1997).

La prevalencia encontrada en el presente estudio fue relativamente baja pero se encontró una amplia diversidad de taxones. La infraestructura y tecnificación en la crianza de los gallos de pelea en comparación con los criadores de aves de traspatio pueden explicar las diferencias encontradas (Mwale y Masika, 2011). En todos los casos, el tipo de suministro de agua, consumo de lombriz de tierra, consumo de insectos y tratamiento antiparasitario fue similar para todas las aves de modo que no se pudo determinar el nivel de riesgo de estas variables.

El 78\% de las instalaciones poseía pisos de tierra; no obstante, esta característica no se encontró significativamente asociada a los parásitos intestinales. La literatura muestra resultados contrastantes para este parámetro. Brown et al. (2006) encontraron mayores prevalencias de Eimeria spp en gallinas ponedoras mantenidas en pisos de tie- rra, mientras que Martínez y Bohórquez (1994) obtuvieron menores tasa de positividad en este tipo de piso. La conducta usual de las aves de corral es escarbar el suelo en busca de invertebrados (insectos, lombrices de tierra), los cuales pueden actuar como hospedadores intermediarios de varias clases de helmintos (Permin et al., 1997b; Ogbaje et al., 2012). La encuesta epidemiológica reveló consumo de insectos en todos los casos y acceso al pastoreo por tiempos variados; sin embargo, solo se encontró asociación significativa a la geofagia $(\mathrm{OR}=2.17$, Cuadro 4), de allí que la limpieza de las jaulas y galpones puede jugar un papel muy relevante al disminuir las posibilidades del contacto de las aves con las heces contaminadas (Calderón, 2005), tal como se encontró en el presente estudio donde las tasas de infección fueron menores en jaulas con limpieza diaria (Cuadro 3).

La frecuencia de lavado de los bebederos/comederos no tuvo asociación significativa con la presencia de parasitismo intestinal en los gallos de pelea. Por contraste, el tipo de bebedero y comedero tuvieron una asociación significativa, donde más del $70 \%$ de los criadores empleaba los de tipo artesanal, los cuales dificultan la limpieza (Marín y Benavides, 2007). Asimismo, se determinó que la mezcla de agua y creolina fue más eficiente en lograr la desinfección de los bebederos y comederos de las aves $(\mathrm{OR}=2.01$, Cuadro 3). Si bien, la limpieza y desinfección se encuentran dentro de los procedimientos de bioseguridad en toda actividad de sanidad animal y la creolina y el cloro son considerados agentes químicos adecuados (De la Sota, 2004; Calderón, 2005), la eficacia de todo desinfectante depende de su concentración, duración de empleo, temperatura y $\mathrm{pH}$ de la solución, y la susceptibilidad del microorganismo a la sustancia desinfectante (De la Sota, 2004; Calderón, 2005). En este sentido, existen quistes y ooquistes de varios taxones de protozoos intestinales que son capaces de permanecer viables ante agentes externos adversos (Aparicio-Rodrigo y Tajada-Alegre, 2007). 


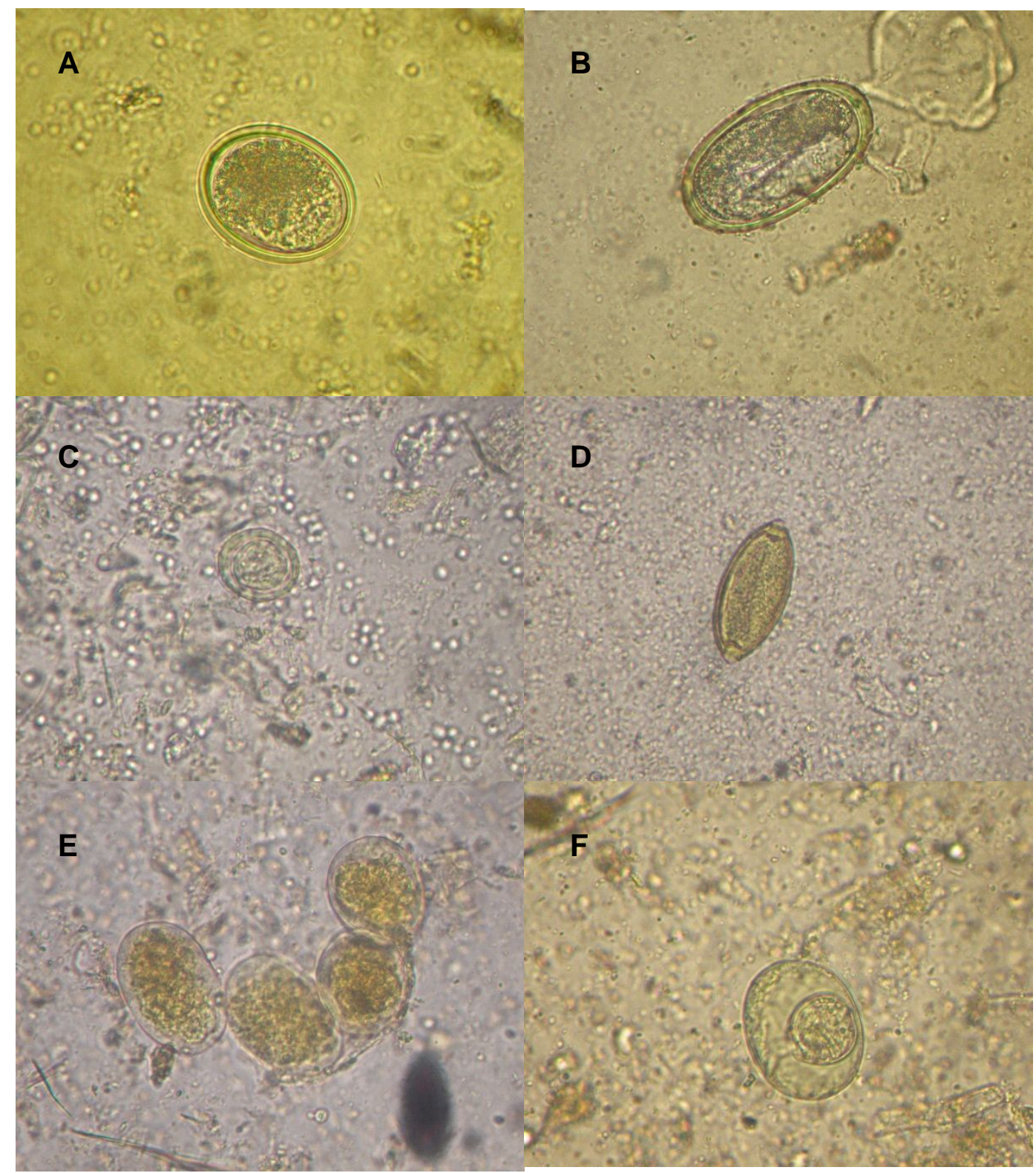

Figura 1. Taxones parasitarios aislados en muestras fecales de gallos de pelea (Gallus gallus domesticus) en la ciudad de Coro, estado Falcón, Venezuela (2012-2013). (a) Huevo de Ascaridia galli, (b) Huevo de Heterakis spp, (c) Huevo de Choanotaenia spp, (d) Huevo de Capillaria spp, (e) Ooquistes de Eimeria spp, (f) Huevo de Raillietina spp, (g). Tinción lugol, 400X 


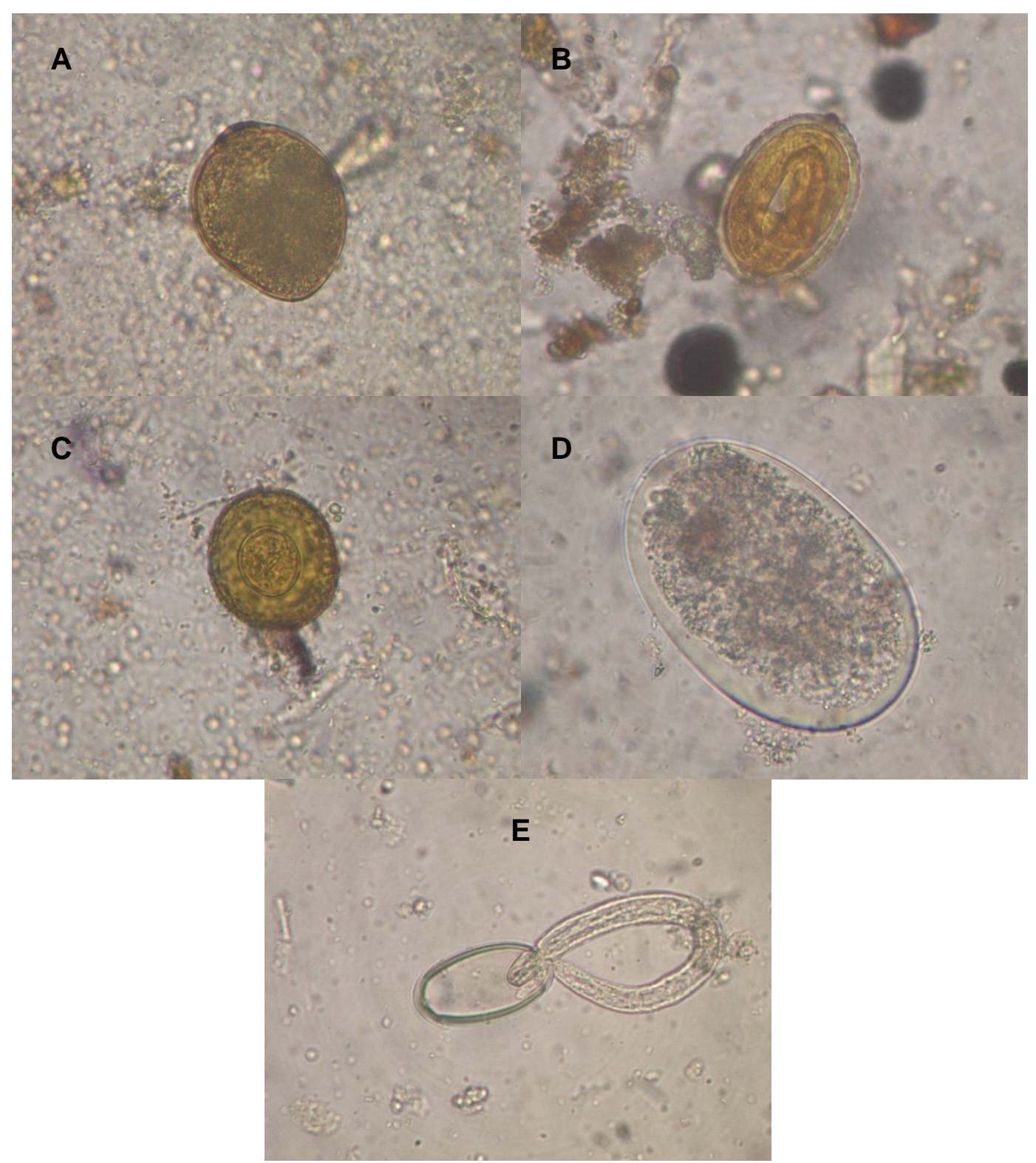

Figura 2. Taxones parasitarios aislados en muestras fecales de gallos de pelea (Gallus gallus domesticus) en la ciudad de Coro, estado Falcón, Venezuela (2012-2013). (a) Huevo de Tetrameres spp, (b) Huevo de Syngamus spp, (c) Huevo de Hymenolepis spp, (d) Huevo de Trichostrogylus spp, (e) Huevo con larva rhabditiforme de Strongyloides spp. Tinción lugol, 400X

La administración adecuada y profesional de drogas antiparasitarias a los gallos de pelea se encuentra dentro de las medidas necesarias para controlar los enteroparásitos y mantener su buen estado de salud, lo que contribuye a un mayor rendimiento en las li- dias (Ibarra-Velarde et al., 2011). Sin embargo, si bien todos los criadores manifestaron que aplicaban tratamiento antiparasitario a las aves, la mayoría sin prescripción del Médico Veterinario, se encontró altas tasas de infección parasitaria en aquellas tratadas 
Cuadro 2. Asociación entre parásitos intestinales en gallos de pelea en la ciudad de Coro, estado Falcón, Venezuela (2012-2013)

\begin{tabular}{|c|c|c|}
\hline \multirow{2}{*}{ Taxones } & \multicolumn{2}{|c|}{ Aves parasitadas } \\
\hline & $\mathrm{n}$ & $\%^{1}$ \\
\hline \multicolumn{3}{|l|}{ Monoparasitismo } \\
\hline Strongyloides spp & 4 & 10.5 \\
\hline Capillaria spp & 2 & 5.4 \\
\hline Choanotaenia spp & 1 & 2.6 \\
\hline Ascaridia galli & 1 & 2.6 \\
\hline Raillietinia spp & 1 & 2.6 \\
\hline \multicolumn{3}{|l|}{ Biparasitismo } \\
\hline Eimeria spp / Choanotaenia spp & 1 & 2.6 \\
\hline Eimeria spp / A. galli & 1 & 2.6 \\
\hline Choanotaenia spp / Capillaria spp & 2 & 5.3 \\
\hline Choanotaenia spp / Strongyloides spp & 4 & 10.5 \\
\hline Choanotaenia spp / Trichostrongylus spp & 1 & 2.6 \\
\hline Heterakis spp /A. galli & 1 & 2.6 \\
\hline Heterakis spp / Capillaria spp. & 1 & 2.6 \\
\hline Strongyloides spp / Capillaria spp & 3 & 7.9 \\
\hline Strongyloides spp / A. galli & 1 & 2.6 \\
\hline Strongyloides $\mathrm{spp} /$ Trichostrongylus spp & 1 & 2.6 \\
\hline \multicolumn{3}{|l|}{ Triparasitismo } \\
\hline Choanotaenia spp / Eimeria spp / Raillietinia spp & 1 & 2.6 \\
\hline Choanotaenia spp / Strongyloides spp / A. galli & 1 & 2.6 \\
\hline Choanotaenia spp / Strongyloides spp / Heterakis spp & 1 & 2.6 \\
\hline Strongyloides spp / Heterakis spp / A. galli & 1 & 2.6 \\
\hline Strongyloides spp / Heterakis spp / Raillietinia spp & 1 & 2.6 \\
\hline Strongyloides spp / Syngamus spp / Capillaria spp & 1 & 2.6 \\
\hline Strongyloides spp / Syngamus spp / Trichostrongylus spp & 1 & 2.6 \\
\hline \multicolumn{3}{|l|}{ Tetraparasitismo } \\
\hline $\begin{array}{l}\text { Capillaria } \mathrm{spp} / \text { Eimeria } \mathrm{spp} / \text { Tetrameres spp / } \\
\text { Trichostrongylus spp }\end{array}$ & 1 & 2.6 \\
\hline $\begin{array}{l}\text { Capillaria spp / Strongyloides spp / Eimeria spp / } \\
\text { Hymenolepis spp }\end{array}$ & 1 & 2.6 \\
\hline $\begin{array}{l}\text { Capillaria spp / Raillietinia spp / Eimeria spp / } \\
\text { Hymenolepis spp }\end{array}$ & 1 & 2.6 \\
\hline $\begin{array}{l}\text { Capillaria } \mathrm{spp} / \text { Eimeria } \mathrm{spp} / \text { Heterakis spp / } \\
\text { Tetrameres } \mathrm{spp}\end{array}$ & 1 & 2.6 \\
\hline \multicolumn{3}{|l|}{ Pentaparasitismo } \\
\hline $\begin{array}{l}\text { Capillaria } \mathrm{spp} / \text { Eimeria } \mathrm{spp} / \text { Strongyloides spp / } \\
\text { Choanotaenia } \mathrm{spp} / \text { Tetrameres } \mathrm{spp}\end{array}$ & 1 & 2.6 \\
\hline $\begin{array}{l}\text { Heterakis } \mathrm{spp} \text { / Strongyloides spp / Capillaria } \mathrm{spp} / \\
\text { Hymenolepis } \mathrm{spp} / \text { Raillietinia } \mathrm{spp}\end{array}$ & 1 & 2.6 \\
\hline $\begin{array}{l}\text { Heterakis spp / Choanotaenia spp / Eimeria spp / } \\
\text { Strongyloides spp / A. galli }\end{array}$ & 1 & 2.6 \\
\hline \multicolumn{3}{|l|}{ Hexaparasitismo } \\
\hline $\begin{array}{l}\text { Heterakis spp / Strongyloides spp / Capillaria spp / } \\
\text { Hymenolepis spp / Raillietinia } \mathrm{spp} / \text { Tetrameres spp }\end{array}$ & 1 & 2.6 \\
\hline
\end{tabular}

${ }^{1}$ En base a 38 aves infectadas 
Cuadro 3. Características de mantenimiento e higiene de jaulas y su asociación con parásitos entéricos de gallos de pelea, Coro, estado Falcón, Venezuela (2012-2013)

\begin{tabular}{|c|c|c|c|c|}
\hline \multirow{2}{*}{ Variable } & \multirow{2}{*}{$\begin{array}{l}\text { Total de aves } \\
\text { (n) }\end{array}$} & \multicolumn{2}{|c|}{ Aves parasitadas } & \multirow{2}{*}{$\mathrm{p}$} \\
\hline & & $\mathrm{n}$ & $\%$ & \\
\hline \multicolumn{5}{|l|}{ Piso } \\
\hline Tierra & 80 & 29 & 36.3 & \multirow{2}{*}{0.69} \\
\hline Cemento & 22 & 9 & 40.9 & \\
\hline \multicolumn{5}{|l|}{ Suministro de agua } \\
\hline Tubería & 102 & 38 & 37.3 & \\
\hline Tanque/tonel & 0 & 0 & 0.0 & \\
\hline \multicolumn{5}{|l|}{ Frecuencia de limpieza } \\
\hline Diario & 30 & 17 & 56.7 & \multirow[b]{2}{*}{0.009} \\
\hline 1 o 2 veces por semana & 72 & 21 & 29.2 & \\
\hline \multicolumn{5}{|l|}{ Tipo de bebederos / comederos } \\
\hline Comercial & 30 & 16 & 53.3 & \multirow[t]{2}{*}{0.01} \\
\hline Artesanal & 72 & 22 & 30.8 & \\
\hline \multicolumn{5}{|c|}{$\begin{array}{l}\text { Frecuencia de lavado de bebederos/ } \\
\text { comederos }\end{array}$} \\
\hline Diario & 34 & 11 & 32.4 & \multirow{2}{*}{0.47} \\
\hline 2 o 3 veces por semana & 68 & 27 & 39.7 & \\
\hline \multicolumn{5}{|l|}{$\begin{array}{l}\text { Desinfectante de bebederos / } \\
\text { comederos }\end{array}$} \\
\hline Agua + cloro & 32 & 17 & 53.1 & \multirow[t]{2}{*}{0.03} \\
\hline Agua + creolina & 70 & 21 & 30.0 & \\
\hline
\end{tabular}

con asistencia veterinaria profesional, lo que sugiere que otros factores como las condiciones de mantenimiento y sanitarias pudieran estar jugando un papel más preponderante en la dinámica de trasmisión.

Se debe resaltar el $20.6 \%$ de prevalencia de Strongyloides spp en el presente estudio; especialmente debido a su magnitud y a que no había sido reportado en el estado Falcón (Duno y Sambrano, 1997; Jiménez y Pereira, 2012). Este resultado es menor al $75 \%$ detectado en aves de traspatio en Nicaragua (Luna-Olivares et al., 2006) y al 5368\% en Malasia (Rahman et al., 2009), aunque mayor a otros reportes que mencionan tasas de prevalencia entre 2 y $10 \%$ (Poulsen et al., 2000; Irungu et al., 2004). En infecciones intensas, especialmente en aves juveniles, estos helmintos pueden ocasionar emaciación con diarrea sanguinolenta (Kaufmann, 1996).

Capillaria spp tampoco había sido reportado en aves en la zona del estudio (Duno y Sambrano, 1997; Jiménez y Pereira, 2012), donde el $16.7 \%$ de prevalencia en los gallos de pelea fue menor al $25 \%$ encontrado por Marín y Benavides (2007) y al $100 \%$ reportado por Percy et al. (2012) en aves de traspatio. No obstante, es una frecuencia mayor al $1.5 \%$ detectado por Irungu et al. (2004). Algunas especies de este género requieren de lombrices de tierra (anélidos) como 
Cuadro 4. Características de la alimentación de gallos de pelea y su asociación con parásitos entéricos, Coro, estado Falcón, Venezuela (2012-2013)

\begin{tabular}{lcccc}
\hline \multirow{2}{*}{ Variable } & $\begin{array}{c}\text { Total de aves } \\
(\mathrm{n})\end{array}$ & \multicolumn{2}{c}{ Aves parasitadas } & $\mathrm{p}$ \\
\cline { 3 - 3 } & & $\mathrm{n}$ & $\%$ & \\
\hline Tiempo de pastoreo (min) & 80 & 29 & 36.3 & \\
$\quad$ 10 a 30 & 22 & 9 & 40.9 & 0.69 \\
31 a 60 & & & & \\
Frecuencia de pastoreo & 34 & 11 & 32.4 & \multirow{2}{*}{0.47} \\
$\quad$ Diario & 68 & 27 & 39.7 & \\
$\quad$ 1 o 2 veces por semana & & & & \\
Geofagia & 52 & 26 & 50.0 & \multirow{2}{*}{0.007} \\
$\quad$ Sí & 50 & 12 & 24.0 & \\
$\quad$ No & & & & \\
Consumo de lombriz de tierra & 0 & 0 & 0.0 & \\
$\quad$ Sí & 102 & 38 & 37.3 & \\
$\quad$ No & & & & \\
Consumo de insectos & 102 & 38 & 37.3 & \\
$\quad$ Sí & 0 & 0 & 0.0 & \\
$\quad$ No & & & & \\
\hline
\end{tabular}

Cuadro 5. Tratamiento antiparasitario y asesoría veterinaria en granjas de gallos de pelea y su asociación con parásitos entéricos, Coro, estado Falcón, Venezuela (20122013)

\begin{tabular}{lcccc}
\hline \multirow{2}{*}{ Variable } & $\begin{array}{c}\text { Total de aves } \\
(\mathrm{n})\end{array}$ & \multicolumn{2}{c}{ Aves parasitadas } & \multirow{2}{*}{$\mathrm{p}$} \\
\cline { 3 - 4 } & & $\mathrm{n}$ & $\%$ & \\
\hline Tratamiento antiparasitario & 102 & 38 & 37.3 & \\
$\quad$ Sí & 0 & 0 & 0.0 & \\
$\quad$ No & & & & \\
Frecuencia de tratamiento & 30 & 17 & 56.7 & \multirow{2}{*}{0.009} \\
$\quad$ 21 a 30 días & 72 & 21 & 29.2 & \\
$\quad$ >30 días & & & & \\
Prescripción del antiparasitario & 18 & 15 & 83.3 & \multirow{2}{*}{0.000} \\
$\quad$ Médico Veterinario & 84 & 23 & 27.4 & \\
$\quad$ Criador & & & & \\
\hline
\end{tabular}


hospedadores intermediarios en su ciclo biológico de allí que llama la atención que los criadores hayan indicado que sus aves no consumían a estos invertebrados. Los nematodos de este género parasitan especialmente el buche y esófago de aves domésticas, pudiendo ocasionar inflamación catarral y diftérica, con adelgazamiento de las paredes de estos órganos, con la consiguiente pérdida de peso, especialmente cuando se tienen altas cargas parasitarias (Kaufmann, 1996; Marín y Benavides, 2007).

Los áscaris Heterakis spp y Ascaridia galli son los más frecuentes en $G$. $g$. domesticus criados en forma libre. Los adultos de Heterakis spp habitan en el ciego, y en infecciones severas pueden producir inflamación y adelgazamiento de la pared intestinal. El efecto más llamativo de este helminto radica en que sus huevos pueden actuar como transportadores del protozoario Histomonas meleagridis, agente etiológico de la histomoniasis (Kaufmann, 1996; Permin et al., 1997a,b). La infección por A. galli, cuyos adultos se alojan en el intestino delgado, pueden producir irritación de la mucosa con hemorragia, causando anemia con limitado desarrollo pondo-estatural (Kaufmann, 1996; Permin et al., 1997a,b). Las frecuencias observadas en los gallos de pelea (Cuadro 2) son similares al registro de $5.2 \%$ de $A$. galli en gallinas ponedoras del estado venezolano de Trujillo (Brown et al., 2006), aunque inferiores a las tasas de prevalencia encontradas en el estado Falcón (Duno y Sambrano, 1997; Jiménez y Pereira, 2012), y en Colombia (Marín y Benavides, 2007) y Nigeria (Ogbaje et al., 2012).

Las prevalencias de Syngamus spp (2\%) y Trichostrongylus spp (3.9\%), coinciden con otros trabajos en el país (Mayaudon, 1955; Luka y Ndams, 2007; Luna-Olivares et al., 2006) y en otras latitudes (Mwale y Masika, 2011). Syngamus spp y particularmente $S$. trachea, son nematodos hematófagos que habitan en el árbol respiratorio de las aves, pudiendo ocasionar obstrucción de la tráquea y asfixia (Kaufmann, 1996). Trichostrongylus spp son nematodos muy patógenos, particularmente $T$. tenuis, que habitan mayormente en el ciego de las aves, pudiendo causar anemia con inapetencia y emaciación cuando se presentan cargas parasitarias altas (Kaufmann, 1996). Por otro lado, Tetrameres spp, cuya trasmisión se encuentra estrechamente relacionada con insectos como cucarachas y saltamontes, presentó una baja prevalencia (Cuadro 2), similar a otros estudios (Percy et al., 2012). Los estadios adultos de estos helmintos habitan principalmente a nivel del proventrículo y esófago (Kaufmann, 1996).

La eimeriosis es una coccidiosis que ocasiona grandes pérdidas económicas en la cría de aves (Williams, 1999), y que ocasiona diarrea, malabsorción y elevada mortalidad (Dalloul y Lillehoj, 2006). El 4.9\% de prevalencia encontrada en el presente estudio fue bastante bajo en comparación con otros estudios en gallinas o pollos de engorde, donde se reportan frecuencias de 17 a $51 \%$ (Martínez y Bohórquez, 1994; Tamasaukas et al., 1998; Brown et al., 2006). El hacinamiento es uno de los factores que contribuyen a la aparición de estos protozoarios (Williams, 1999; Dalloul y Lillehoj, 2006), por lo que posiblemente el confinamiento en solitario de los gallos de pelea haya disminuido la propagación de los ooquistes de Eimeria spp.

\section{Conclusiones}

La prevalencia de parásitos intestinales en gallos de pelea en la zona de Coro, estado Falcón, Venezuela fue de $37.3 \%$.

\section{Literatura Citada}

1. Álvarez C, Rodríguez P, Carvajal E. 2011. Efecto del extracto de paico (Chenopodium ambrosioides), en parásitos gastrointestinales de gallos de pelea (Gallus domesticus). Cultura Científ 9: 76-80. 
2. Aparicio-Rodrigo M, Tajada-Alegre P. 2007. Parasitosis intestinales. Pediatr Integr 11: 149-160.

3. Benjoya J. 1972. Prevalencia de helmintos parásitos del intestino de gallinas (Gallus domesticus), provenientes del estado Aragua, Tesis de Médico Veterinario. Maracay: Univ Central de Venezuela. $37 \mathrm{p}$.

4. Botero D, Restrepo M. 2003. Parasitosis intestinales por helmintos. En: Parasitosis humanas. $4^{\circ} \mathrm{ed}$. Colombia: Corporación para Investigaciones Biológicas. p 93-160.

5. Brown E, Díaz D, Moreno L, Gotopo A. 2006. Prevalencia de Eimeria spp en gallinas ponedoras de granjas pertenecientes a tres municipios del estado Trujillo, Venezuela. Rev Científ FCVLUZ 26: 579-584.

6. Calderón K. 2005. Comparación del efecto desinfectante de hidróxido de calcio vs. una mezcla de ácidos orgánicos y surfactantes, aplicados en pediluvios de una granja avícola en el departamento de Guatemala. Tesis de Médico Veterinario. Guatemala: Univ San Carlos de Guatemala. 51 p.

7. Chadfield M, Permin A, Nansen $P$, Bisgaard M. 2001. Investigation of the parasitic nematode Ascaridia galli (Shrank 1788) as a potential vector for Salmonella enterica dissemination in poultry. Parasitol Res 87:317-325.

8. Dalloul R, Lillehoj H. 2006. Poultry coccidiosis: recent advancements in control measures and vaccine development. Expert Rev Vaccines 5: 143-163.

9. De la Sota M. 2004. Manual de procedimientos de desinfección. Argentina: Servicio Nacional de Sanidad y Calidad Agroalimentaria. $28 \mathrm{p}$.

10. Duno A, Sambrano G. 1997. Determinación de helmintos en aves de combate (Gallus domesticus) en el municipio autónomo Miranda. Tesis de Médico Veterinario. Coro: Univ Nacional Experimental Francisco de Miranda. 35 p.
11. Eslami A, Ghaemi P, Rahbari S. 2009. Parasitic infections of free-ranged chickens from Golestan province, Iran. Iran J Parasitol 4: 10-14.

12. Eshetu Y, Mulualem E, Ibrahim H, Berhanu A, Aberra K. 2001. Study of gastro-intestinal helminths of scavenging chickens in four rural districts of Amhara region, Ethiopia. Rev Sci Tech Off Int Epiz 20: 791-796.

13. Ewel J, Madriz A, Tosi Jr J. 1976. Monte espinoso tropical. En: Zonas de vida de Venezuela. Memoria explicativa sobre el mapa ecológico. $4^{\circ}$ ed. Venezuela: Ed Sucre. p 56-65.

14. Fink M, Permin A, Magwisha H, Jensen K. 2005. Prevalence of the proventricular nematode Tetrameres americana Cram (1927) in different age groups of chickens in the Morogoro region, Tanzania. Trop Anim Health Prod 37: 133-137.

15. Gomes F, Machado H, Lemos L, Almeida L, Daher R. 2009. Principais parasitos intestinais diagnosticados em galinhas domésticas criadas em regime extensivo na municipalidade de Campos dos Goytacazes, RJ. Ciênc Anim Bras 10:818-822.

16. Ibarra-Velarde F, Guerrero-Molina C, Vera-Montenegro Y, Alcalá-Canto Y, Romero-Callejas E. 2011. Comparison of the anthelmintic efficacy of three commercial products against ascarids and Capillaria spp. in fighting cocks. J Pharm Pharmacol 2: 146-150.

17. Irungu L, Kimani R, Kisia S. 2004. Helminth parasites in the intestinal tract of indigenous poultry in parts of Kenya. J S Afr Vet Assoc 75: 58-59.

18. Jiménez P, Pereira D. 2012. Helmintos gastrointestinales en gallinas de traspatio sector Turupía, municipio Zamora del estado Falcón. Tesis de Médico Veterinario. Coro: Univ Nacional Experimental Francisco de Miranda. 39 p.

19. Kaufmann J. 1996. Parasites of poultry. In: Parasitic infections of domestic 
animals: a diagnostic manual. Germany: Basel Birkhäuser Verlag. p 337-393.

20. Luka S, Ndams I. 2007. Gastrointestinal parasites of domestic chicken Gallus gallus domesticus Linneaeus 1758 in Samaru, Zaria, Nigeria. Sci World J 2: 27-29.

21. Luna Olivares L, Kyvsgaard $N$, Rimbaud E, Pineda N. 2006. Prevalencia y carga parasitaria de helmintos gastrointestinales en gallinas de traspatio (Gallus gallus domesticus), en el municipio de El Sauce, departamento de León, Nicaragua. Redvet 7: 1-4.

22. Marín S, Benavides J. 2007. Parásitos en aves domésticas (Gallus domesticus) en el Noroccidente de Colombia. Vet Zootec 1: 43-51.

23. Martínez de CH, Bohórquez N. 1994. Prevalencias y factores asociados a la coccidiosis en pollos de engorde. Rev Científ FCV-LUZ 4: 25-36.

24. Mayaudon H. 1955. Endoparásitos de las gallinas (Gallus gallus domesticus) en Venezuela. Rev Med Vet Parasitol 14: 99-122.

25. Mayaudon H, Montoya F. 1970. Contribución al estudio de la fauna parasitaria de las aves de Venezuela. Rev Med Vet Parasitol 13: 227-230.

26. Mwale M, Masika P. 2011. Point prevalence study of gastro-intestinal parasites in village chickens of Centane district, South Africa. Afr J Agric Res 6: 2033-2038.

27. Morales G, Pino de Morales L. 1977. Coprología. En: Manual de diagnóstico helmintológico en rumiantes. Venezuela: Colegio de Médicos Veterinarios del Estado Aragua. p 26-27.

28. Morales G, Pino de Morales L. 1995. Conceptos básicos en ecología y epidemiología de parásitos. En: Parasitometría. Venezuela: Clemente Ed. p 27-53.

29. Ogbaje C, Agbo E, Ajanusi O. 2012. Prevalence of Ascaridia galli, Heterakis gallinarum and Tapeworm infections in birds slaughtered in Makurdi township. Int J Poult Sci 11: 103-107.
30. Oniye S, Audu P, Adebote D, Kwaghe $B$, Ajanusi O, Nfor M. 2000. Survey of helminth parasites of laughing dove (Streptopelia senegalensis) in Zaria Nigeria. Afr J Nat Sci 4: 65-66.

31. Panja A, Siden E, Mayer L. 1995. Synthesis and regulation of accessory/ proinflammatory cytokines by intestinal epithelial cells. Clin Exp Immunol 100: 298-305.

32. Paul D, Dey A, Bilkis F, Begum N, Mondal M. 2012. Epidemiology and pathology of intestinal helminthiasis in fowls. Eurasian J Vet Sci 28: 31-37.

33. Percy J, Pias M, Desberia B, Lucia T. 2012. Seasonality of parasitism in free range chickens from a selected ward of a rural district in Zimbabwe. Afr J Agric Res 7: 3626-3631.

34. Permin A, Bojesen M, Nansen P, Bisgaard M, Frandsen F, Pearman M. 1997a. Ascaridia galli populations in chickens following single infections with different dose levels. Parasitol Res 83: 614-617.

35. Permin A, Magwisha H, Kassuku A, Nansen P, Biggaard M, Frandsen F, Gibbons L. 1997b. A cross-sectional study of helminths in rural scavenging poultry in Tanzania in relation to season and climate. J Helminthol 71: 233-240.

36. Poulsen J, Permin A, Hindsbo O, Yelifari L, Nansen P, Loch P. 2000. Prevalence and distribution of gastrointestinal helminths and haemoparasites in young scavenging chickens in upper eastern region of Ghana, West Africa. Prev Vet Med 45: 237-245.

37. Rahman A, Salim H, Ghause M. 2009. Helminthic parasites of scaven-ging chickens (Gallus domesticus) from villages in Penang island, Malaysia. Trop Life Sci Res 20: 1-6.

38. Rodríguez R, Cob-Galera L, Domínguez J. 2001. Frecuencia de parásitos gastrointestinales en animales domésticos diagnosticados en Yucatán, México. Rev Biomed 12: 19-25. 
39. Tamasaukas R, Ruiz H, Roa N. 1998. Relación costo-beneficio de la profilaxis de la coccidiosis aviar. Rev Científ FCVLUZ 8: 217-221.

40. Varela A. 2007. Manejo productivo del gallo de pelea (Gallus gallus). Monografía profesional de Médico Veterina- rio Zootecnista. México: Universidad Veracruzana. $44 \mathrm{p}$.

41. Williams R. 1999. A compartmentalized model for the estimation of the cost of coccidiosis to the world's chicken production industry. Int J Parasitol 29: 1209-1229. 